\title{
Microbiology Susceptibility Non-host Organism Identifier
}

National Cancer Institute

\section{Source}

National Cancer Institute. Microbiology Susceptibility Non-host Organism Identifier. NCI

Thesaurus. Code C162145.

Sponsor-defined identifier for a non-host organism. 